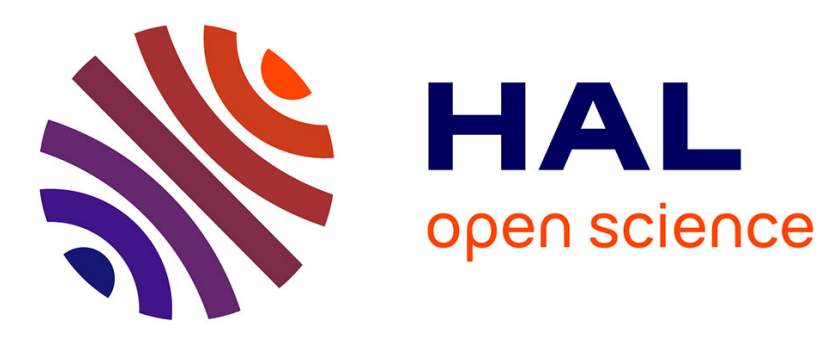

\title{
Motion Control for Steerable Wheeled Mobile Manipulation
}

Mohamed Sorour, Andrea Cherubini, Philippe Fraisse

\section{To cite this version:}

Mohamed Sorour, Andrea Cherubini, Philippe Fraisse. Motion Control for Steerable Wheeled Mobile Manipulation. 2019. hal-02189411

\section{HAL Id: hal-02189411 \\ https://hal.science/hal-02189411}

Preprint submitted on 19 Jul 2019

HAL is a multi-disciplinary open access archive for the deposit and dissemination of scientific research documents, whether they are published or not. The documents may come from teaching and research institutions in France or abroad, or from public or private research centers.
L'archive ouverte pluridisciplinaire HAL, est destinée au dépôt et à la diffusion de documents scientifiques de niveau recherche, publiés ou non, émanant des établissements d'enseignement et de recherche français ou étrangers, des laboratoires publics ou privés. 


\title{
Motion Control for Steerable Wheeled Mobile Manipulation
}

\author{
Mohamed Sorour $^{1}$, Andrea Cherubini ${ }^{2}$, and Philippe Fraisse ${ }^{2}$
}

\begin{abstract}
In this paper, we address the problem of long travel mobile manipulation for steerable wheeled mobile robots (SWMR) operating in human shared environment. On one hand, a small footprint is required while maintaining a fixed arm configuration, to make robot motion predictable for near individuals during the long traverse. On the other hand, redundancy resolution poses a challenge since there is no direct kinematic mapping between the task and joint spaces for SWMR. Hence, we propose a redundancy resolution algorithm that enables switching between 3 modes of operation based on the Euclidean norm of the motion task error. In particular, we employ a floating base model for the mobile platform, and enhance the end effector motion performance by predicting the error between such model and the actual (SWMR) one. Such error is then compensated using the highly responsive arm manipulator. The proposed methodology is successfully validated in simulations on a Neobotix-MPO700 SWMR with a Kuka LWR-IV manipulator mounted on it.

Index Terms-mobile manipulation, pseudo-omni mobile robot, steerable mobile robot, long travel manipulation.
\end{abstract}

\section{INTRODUCTION}

Mobile manipulators employing steerable wheeled mobile robots (SWMR) have better workspace reachability than most other types of mobile robots. The SWMR structure is able to perform complex $2 D$ planar trajectories despite its non-holonomic constraints. However, redundancy resolution and motion control approaches commonly used in the literature [1]-[3] comprising extended (or augmented) Jacobian matrices for the mobile base and the arm manipulator will not result in acceptable end-tip motion performance. The reason is that for SWMR, there is no direct kinematics relation (the Jacobian) between actuation commands and operational space velocities. This is in fact the case for all mobile robot structures with 1 or more degrees of steerability, and was only treated in [4] via input-output/dynamic feedback linearization. However, such method is only suitable for differential drive systems, for which task space velocities can be mapped to joint rates. In addition, the kinematic models used in the literature are either too generic (assuming a fully omni-directional mobile base) [5]-[7], or only specific for one particular mobile robot structure, most commonly: differential drive [8], [9], unicycle [10], four-wheel drive [11][13], or car-like [1]. None exist for steerable wheeled mobile robots.

Moreover, motion control based on the extended mobile manipulation Jacobian matrix will result in mobile-base/arm motion at all times. Such behavior is non desirable in plenty of applications due to the different tasks assigned to the mobile base and/or to the manipulator. Usually, the mobile

\footnotetext{
${ }^{1}$ Lincoln Center for Autonomous Systems (L-CAS), School of Computer Science, University of Lincoln, Brayford Pool, LN6 7TS Lincoln, United Kingdom. msorouralincoln.ac.uk

${ }^{2}$ LIRMM, Université de Montpellier, CNRS, Montpellier, France. firstname.lastnamedirmm.fr
}

base gives extended workspace at low accuracy due to wheel slippage and lack of absolute position sensing capabilities [14], whereas the arm enables high accuracy manipulation, in a limited workspace. In practice, these two tasks are performed one at a time due to their very different nature. To this end, motion control techniques capable of activating each/both systems according to the task in hand would be more realistic and useful.

On the other hand, maintaining the arm in a folded, dexterous configuration is advantageous during long travel, especially in applications where the robot/human share the same workspace. Usually, this can be done by maximizing the manipulability measure [15] as has been done, for different purposes in [14], [16]-[18]. However such approach does not guarantee a fixed arm configuration (or a range of close configurations), especially if such task is implemented as one of the secondary, lower priority hierarchical tasks [19]. This makes the arm motion, although characterized by a high manipulability index, unpredictable [20] to an individual sharing the same workspace.

In this work, we propose a redundancy resolution formulation that organizes the sharing of the required end-tip motion (pose control application) task among the mobile base and/or the arm. The formulation based on the weighted pseudoinverse [21], employing a variation of the approach used in [22], allows switching between 3 modes of operation:

- Gross Motion: only the base is responsible for performing end-tip motion task, while the arm maintains a predefined arbitrary dexterous configuration. This mode is active if long workspace travel is necessary.

- Mobile Manipulation: both the arm and mobile base participate in the motion task, when the end-tip is reasonably close to its target.

- Fine Manipulation: the motion task is mainly done by the arm. Meanwhile, the mobile base motion is kept minimal except for achieving/maintaining the dexterous configuration. This mode is activated if the end-tip is very close to its target.

To accommodate for the steerable mobile robot complex structure, we compensate the expected error in the mobile robot motion by the highly accurate/dynamic arm manipulator. Such compensation is possible thanks to the SWMR controller developed in previous work [23], [24]. There, the feasible "next sample period" robot velocity can be computed based on the joint performance limits of the SWMR hardware, while taking into account its nonholonomic constraints. We summarize the contributions of this work as follows:

1) Redundancy resolution formulation most convenient for long travel mobile manipulation in human shared environment. 
2) Such formulation takes into account steerable wheeled mobile robot performance limits, predicts and compensates its motion errors.

In the following, section II introduces the general formulation of the problem in hand. The proposed redundancy resolution method is detailed in section III. A brief description of the steerable mobile base controller is presented in section IV. Simulations are depicted in section V. Conclusions are finally given in section VI.

\section{Problem Formulation}

Let the configuration space coordinates of the mobile manipulator be $\overline{\mathbf{q}}=\left[\begin{array}{ll}\overline{\mathbf{q}}_{b}^{\top} & \mathbf{q}_{a}^{\top}\end{array}\right]^{\top}$, where $\mathbf{q}_{a} \in \mathbb{R}^{n_{a}}$ are the generalized coordinates of the arm manipulator, and $\overline{\mathbf{q}}_{b} \in$ $\mathbb{R}^{n_{b}}$ is a reduced order representation of the configuration space of the SWMR, constituted of only the $6 D$ robot pose. Despite assuming the mobile robot treated in this work is a floating base operating in the $2 D$ planar workspace, we use $n_{b}=6$ for the purpose of proper mathematical development. The links and joints of the mobile manipulator are assumed perfectly rigid, and the arm is a fully actuated open serial chain. The respective configuration vectors are detailed as:

$$
\begin{aligned}
& \mathbf{q}_{a}=\left[\begin{array}{lll}
q_{1} & \ldots & q_{n_{a}}
\end{array}\right]^{\top}, \\
& \overline{\mathbf{q}}_{b}=\left[\begin{array}{llll}
x & y & \mathbf{0}_{1 \times 3} & \alpha
\end{array}\right]^{\top},
\end{aligned}
$$

where $\alpha$ is the rotation angle about $\mathbf{z}$ axis.

\section{A. End-Tip Velocity Control}

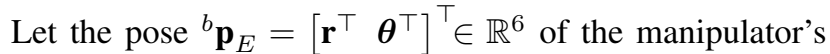
end tip frame $\mathcal{F}_{E}$ expressed in the base frame $\mathcal{F}_{b}$ define the task space coordinates (the respective frames are depicted in Fig. 1), with $\mathbf{r}=\left[\begin{array}{lll}x & y & z\end{array}\right]^{\top}$ being the position vector, and $\boldsymbol{\theta}=\left[\begin{array}{lll}\gamma & \beta & \alpha\end{array}\right]^{\top}$ denoting a minimal representation of orientation (roll, pitch, yaw RPY variation of Euler angles). The physical velocity vector is given by ${ }^{b} \dot{\mathbf{x}}_{E}=\left[\begin{array}{ll}\dot{\mathbf{r}}^{\top} & \boldsymbol{\omega}^{\top}\end{array}\right]^{\top} \in$ $\mathbb{R}^{6}$. In practice, the readily available kinematic mapping (the Jacobian) from the configuration space to the task space for the arm is given by:

$$
{ }^{n_{a}} \dot{\mathbf{x}}_{n_{a}}^{0}=\mathbf{J}_{n_{a}} \dot{\mathbf{q}}_{a}
$$

relating the velocity of the final link frame $\mathcal{F}_{n_{a}}$ w.r.t the initial link frame $\mathcal{F}_{0}$ expressed in $\mathcal{F}_{n_{a}}$ with the joint space velocity vector $\dot{\mathbf{q}}_{a}$. Note that from the task definition above, $\mathbf{J}_{n_{a}}$ is the $6 \times n_{a}$ geometric Jacobian matrix. The end tip frame is related to the last frame by a constant homogeneous transformation matrix ${ }^{n_{a}} \mathbf{T}_{E}$, and so is the initial frame to the mobile base frame ${ }^{b} \mathbf{T}_{0}$. Then, the contribution of the arm manipulator to the velocity of the end tip, expressed in the base frame, can be obtained using:

$$
\begin{gathered}
{ }^{b} \dot{\mathbf{x}}_{E(a)}=\mathbf{J}_{a} \dot{\mathbf{q}}_{a}, \\
\mathbf{J}_{a}={ }^{b} \mathbb{T}_{E}{ }^{E} \mathbb{T}_{n_{a}} \mathbf{J}_{n_{a}},
\end{gathered}
$$

with ${ }^{b} \mathbb{T}_{E}$, and ${ }^{E} \mathbb{T}_{n_{a}}$ the spatial transformation matrices:

$$
{ }^{E} \mathbb{T}_{n_{a}}=\left[\begin{array}{cc}
n_{a} \mathbf{R}_{E}^{\top} & { }^{n_{a}} \mathbf{R}_{E}^{\top} \times{ }^{n_{a}} \mathbf{r}_{E} \\
\mathbf{0}_{3} & n_{a} \mathbf{R}_{E}^{\top}
\end{array}\right]
$$

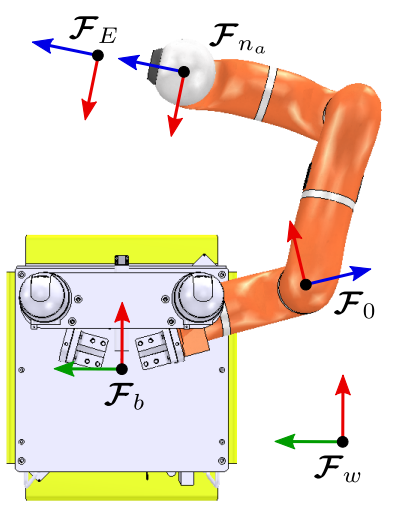

Fig. 1: Mobile manipulator frames used in our development, $\mathbf{x}, \mathbf{y}$, and $\mathbf{z}$ axes colored in red, green, and blue respectively.

$$
{ }^{b} \mathbb{T}_{E}=\left[\begin{array}{cc}
{ }^{b} \mathbf{R}_{E} & \mathbf{0}_{3} \\
\mathbf{0}_{3} & { }^{b} \mathbf{R}_{E}
\end{array}\right],
$$

${ }^{n_{a}} \mathbf{R}_{E} \in \mathbb{S O}(3)$, and ${ }^{n_{a}} \mathbf{r}_{E}$ being the $3 \times 3$ rotation matrix and $3 \times 1$ position vectors respectively obtained from ${ }^{n_{a}} \mathbf{T}_{E}$. Similarly, ${ }^{b} \mathbf{R}_{E}$ is obtained from ${ }^{b} \mathbf{T}_{E}={ }^{b} \mathbf{T}_{0}{ }^{0} \mathbf{T}_{n_{a}}{ }^{n_{a}} \mathbf{T}_{E}$.

For the mobile robot, we propose a general formulation since the SWMR does not have a usable kinematic (velocity) mapping from the joint space to the task space (in contrast with differential drive systems, for instance). We assume a 3DOF, 3DOM (floating base) mobile robot constituted of 2 prismatic joints along the base frame $\mathbf{x}$ and $\mathbf{y}$ axes, plus 1 revolute joint about the $\mathbf{z}$ axis. In such case, the readily available kinematic mapping is given as:

$$
\begin{aligned}
{ }^{b} \dot{\mathbf{x}}_{b}^{w} & =\mathbf{J}_{b} \dot{\mathbf{q}}_{b}, \\
\mathbf{J}_{b} & =\mathbf{I}_{6},
\end{aligned}
$$

relating the mobile base velocity w.r.t the fixed world frame $\mathcal{F}_{w}$ expressed in $\mathcal{F}_{b}$, where $\mathbf{I}_{6}$ is the $6 D$ identity matrix. Note that:

$$
\dot{\mathbf{q}}_{b}=\left[\begin{array}{llll}
\dot{x} & \dot{y} & \mathbf{0}_{1 \times 3} & \omega_{z}
\end{array}\right]^{\top},
$$

is not the derivative of $\overline{\mathbf{q}}_{b}$. Using (2), we compute the contribution of the mobile base velocity to that of the end effector as:

$$
\begin{gathered}
{ }^{b} \dot{\mathbf{x}}_{E(b)}=\mathbb{H} \mathbf{J}_{b} \dot{\mathbf{q}}_{b} \\
\mathbb{H}=\left[\begin{array}{cccccc}
1 & 0 & 0 & 0 & 0 & -{ }^{b} y_{E} \\
0 & 1 & 0 & 0 & 0 & { }^{b} x_{E} \\
& \mathbf{0}_{3 \times 5} & & \mathbf{0}_{3 \times 1} \\
0 & 0 & 0 & 0 & 0 & 1
\end{array}\right]
\end{gathered}
$$

\section{B. Pose Control}

For pose control applications, we need the analytic Jacobian (orientation representation specific) rather than the Geometric Jacobian in (1). This is computed as:

$$
\begin{gathered}
{ }^{b} \dot{\mathbf{p}}_{E(a)}=\mathbb{M}_{\mathrm{RPY}} \mathbf{J}_{a} \dot{\mathbf{q}}_{a}, \\
{ }^{b} \dot{\mathbf{p}}_{E(b)}=\mathbb{M}_{\mathrm{RPY}} \mathbb{H} \mathbf{J}_{b} \dot{\mathbf{q}}_{b},
\end{gathered}
$$

for the arm and base respectively (i.e., from (1), (4)). Here $\mathbb{M}_{\mathrm{RPY}}$ is the transformation matrix for RPY angles:

$$
\mathbb{M}_{\mathrm{RPY}}=\left[\begin{array}{cc}
\mathbf{I}_{3} & \mathbf{0}_{3} \\
\mathbf{0}_{3} & \boldsymbol{\Omega}_{\mathrm{RPY}}
\end{array}\right],
$$




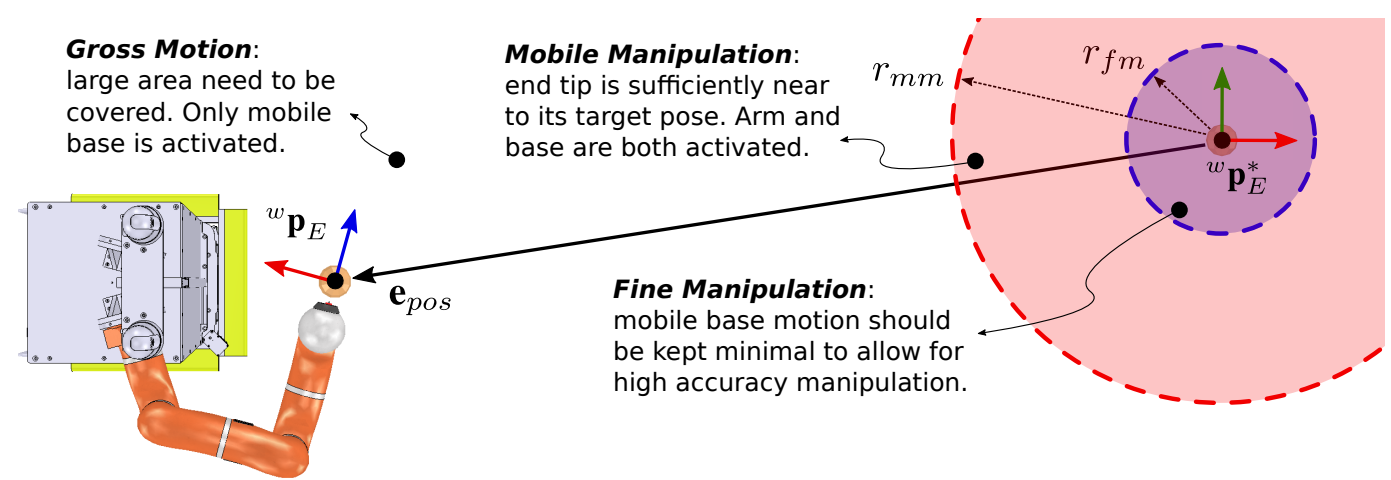

Fig. 2: Mobile manipulation motion control based on pose error.

$$
\boldsymbol{\Omega}_{\mathrm{RPY}}=\left[\begin{array}{ccc}
\cos (\alpha) \cos (\beta) & -\sin (\alpha) & 0 \\
\sin (\alpha) \cos (\beta) & \cos (\alpha) & 0 \\
-\sin (\beta) & 0 & 1
\end{array}\right] .
$$

Since the inverse of $\mathbb{M}_{\mathrm{RPY}}$ is singular at $\beta=\pi / 2$, we use the damped pseudo-inverse [25], [26] of the analytic Jacobian in (5) and (6) for redundancy resolution in the sequel.

\section{REDUndAnCy RESOlUtion}

From (5) and (6), we can construct the augmented kinematic mapping:

$$
{ }^{b} \dot{\mathbf{p}}_{E}=\mathbf{J}_{m m} \dot{\mathbf{q}}
$$

relating the velocity of the proposed joint space coordinates to the end tip velocity expressed in the base frame with:

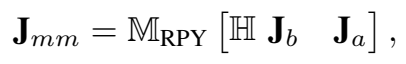

the $6 \times\left(n_{a}+n_{b}\right)$ augmented mobile manipulator Jacobian matrix, and the augmented joint velocity vector given by:

$$
\dot{\mathbf{q}}=\left[\begin{array}{c}
\dot{\mathbf{q}}_{b} \\
\dot{\mathbf{q}}_{a}
\end{array}\right] \text {. }
$$

One possible solution to obtain the joint space velocity $\dot{\mathbf{q}}_{\text {ref }}$ corresponding to a given reference task space velocity (e.g., output by a motion planner) ${ }^{b} \dot{\mathbf{p}}_{E}^{\text {ref }}$, referred to hereafter as $\dot{\mathbf{p}}_{\text {ref }}$ to lighten the notation, is to invert (7):

$$
\dot{\mathbf{q}}_{r e f}=\mathbf{J}_{m m}^{+} \dot{\mathbf{p}}_{r e f} .
$$

Here, $\mathbf{J}_{m m}^{+}$is the Moore-Penrose pseudoinverse of matrix $\mathbf{J}_{m m}$. However, the solution of (8) will always result in moving the arm and the base simultaneously. In practice, such behavior is undesirable. Consider for instance cases where the arm is performing a delicate object manipulation: it is preferable to block the less accurate mobile base. On the other hand, for a gross movement from one pose to another one several meters apart, there is no interest in moving the arm towards such faraway target. On the contrary, it might be desirable to have an arm folded configuration, that is static so that the end tip motion can be "to a large extent" predictable by humans sharing the workspace. To this end, we propose a task sharing redundancy resolution formulated as:

$$
\dot{\mathbf{q}}_{r e f}=\mathbf{J}_{m m(d)}^{+W} \dot{\mathbf{p}}_{r e f}+\lambda_{\text {dex }}\left(\mathbf{I}_{6+n_{a}}-\mathbf{J}_{m m}^{+} \mathbf{J}_{m m}\right) \mathbf{z}_{d e x},
$$

where $\mathbf{z}_{d e x}$ is arbitrary optimization term, $\mathbf{J}_{m m(d)}^{+W}$ is the weighted [21], damped pseudoinverse of $\mathbf{J}_{m m}$, given by:

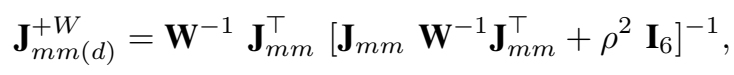

with $\rho$ the damping factor, and $\mathbf{W}$ the $\left(6+n_{a}\right) \times\left(6+n_{a}\right)$ symmetric, positive definite weight matrix:

$$
\mathbf{W}=\left[\begin{array}{cc}
(1-\psi) \mathbf{I}_{6} & \mathbf{0}_{6 \times n_{a}} \\
\mathbf{0}_{n_{a} \times 6} & \psi \mathbf{I}_{n_{a}}
\end{array}\right] .
$$

The parameter $\psi \in[0,1]$ in 10$]$ is the task sharing factor. If $\psi=1$, only the arm is responsible for performing the pose control task, whereas if $\psi=0$, only the base is activated. The value of $\psi$ is computed based on the pose error as depicted in Fig. 2 using:

$$
\psi= \begin{cases}1, & \text { if }\left\|\mathbf{e}_{\text {pos }}\right\|_{2} \leq r_{f m} \\ 0.5, & \text { if } r_{f m}<\left\|\mathbf{e}_{\text {os }}\right\|_{2} \leq r_{m m} \\ 0, & \text { if }\left\|\mathbf{e}_{\text {pos }}\right\|_{2}>r_{m m}\end{cases}
$$

where $\left\|\mathbf{e}_{\text {pos }}\right\|_{2}$ is the Euclidean norm of the end-tip position error $\left[\begin{array}{ll}\mathbf{I}_{3} & \mathbf{0}_{3}\end{array}\right] \mathbf{e}_{p}, r_{f m}, r_{m m} \in \mathbb{R}^{+}$denote the fine and mobile manipulation ranges respectively. In $[9], \dot{\mathbf{p}}_{\text {ref }}$ is the output of the proportional pose controller:

$$
\begin{gathered}
\dot{\mathbf{p}}_{r e f}=\lambda_{p}{ }^{b} \mathbb{T}_{w} \mathbf{e}_{p}, \\
\mathbf{e}_{p}={ }^{w} \mathbf{p}^{*}-{ }^{w} \mathbf{p}, \\
{ }^{b} \mathbb{T}_{w}=\left[\begin{array}{cc}
{ }^{b} \mathbf{R}_{w} & \mathbf{0}_{3} \\
\mathbf{0}_{3} & { }^{b} \mathbf{R}_{w}
\end{array}\right],
\end{gathered}
$$

with $\lambda_{p}$ a positive scalar gain, $\mathbf{e}_{p}$ the $6 D$ pose error, and ${ }^{w} \mathbf{p},{ }^{w} \mathbf{p}^{*}$ respectively the current and desired poses both expressed in the world frame.

The particular solution in 99, will result in the behavior depicted in Fig. 2, where the base/arm contribution to the pose control task is decided based on the end-tip position error. Parameters $r_{f m}, r_{m m}$ defining the different regions can be tuned based on the application. The homogeneous solution (secondary task) proposed in (9) is defined as:

$$
\mathbf{z}_{d e x}=\left[\begin{array}{c}
\mathbf{0}_{6 \times 1} \\
-\left(\mathbf{q}_{a}-\mathbf{q}_{a(\text { dex })}\right)
\end{array}\right] .
$$

The arbitrary vector formulation in $(13)$, when injected in 9 yields a proportional controller that will force the current arm configuration vector $\mathbf{q}_{a}$ towards a predefined dexterous one $\mathbf{q}_{a(d e x)}$ depending on the positive scalar gain $\lambda_{\text {dex }}$. 
To enhance the overall response to the commands resolved using (9), we propose a corrective action to the base velocity error, to be performed by the usually faster, more accurate and dynamic arm, recall $\dot{\mathbf{q}}_{\text {ref }}=\left[\begin{array}{ll}\dot{\mathbf{q}}_{\text {ref(a) }}^{\top} & \dot{\mathbf{q}}_{\text {ref }(b)}^{\top}\end{array}\right]^{\top}$ :

$$
\dot{\mathbf{q}}_{r e f(a)}=\dot{\mathbf{q}}_{r e f(a)}+\mathbf{J}_{a}^{+}\left[\mathbb{M}_{\mathrm{RPY}} \mathbb{H} \mathbf{J}_{b}\left(\dot{\mathbf{q}}_{r e f(b)}-\dot{\mathbf{q}}_{b f}\right)\right],
$$

where $\dot{\mathbf{q}}_{b f}$ is the feasible next sample time base velocity vector depending on the base controller:

$$
\dot{\mathbf{q}}_{b f}=h(\boldsymbol{\beta}, \boldsymbol{\phi}) \dot{\mathbf{q}}_{r e f(b)} .
$$

Here $h(\boldsymbol{\beta}, \boldsymbol{\phi})$ represents the controller for the mobile robot, and vectors $\boldsymbol{\beta}, \boldsymbol{\phi}$ denote the steering and wheel positions, respectively. A brief description of the SWMR controller $h(\boldsymbol{\beta}, \boldsymbol{\phi})$ will be presented in next section.

\section{Steerable Mobile Robot Controller}

In this section, we briefly recall the motion-discontinuity robust controller developed in our previous work [?], [23]. The schematic of a SWMR is shown in Fig. 3 for a 4 wheeled robot. Frames $\mathcal{F}_{I}=\left(o_{I} \mid \boldsymbol{x}_{I}, \boldsymbol{y}_{I}, \boldsymbol{z}_{I}\right), \mathcal{F}_{b}$ denote the inertial and mobile base frames respectively, with origin $o_{b}$ located at the robot geometric center. Frames $\mathcal{F}_{h i}$, and $\mathcal{F}_{s i}$ represent the $i^{t h}$ hip and steer frames with $i=1, \ldots, N$ ( $N$ being the number of wheels). The hip and steer frames share the same origin, with relative orientation $\beta_{i}$ (the steering angle). Frame $\mathcal{F}_{w i}$ is attached to (but not rotating with) the $i^{t h}$ wheel, assigned such that $\boldsymbol{x}_{w i}$ points along the heading of the wheel, which rotates about $\boldsymbol{y}_{w i}$ by the driving angle $\phi_{i}$. In Fig. 3, the task space velocity components are: $\dot{\mathbf{q}}_{\text {ref(b) }}=\left[\begin{array}{ll}\boldsymbol{v}^{\top} & \omega_{z}\end{array}\right]^{\top}$, where $\boldsymbol{v}=\left[\begin{array}{ll}\dot{x} & \dot{y}\end{array}\right]^{\top}, \omega_{z}$ the mobile base linear and angular velocities respectively.

The objective of the framework, whose block diagram is shown in Fig. 4 is to fulfill the reference mobile base velocity: $\dot{\mathbf{q}}_{\text {ref(b) }}$ (as input) as much as possible, while respecting the maximum steer joint performance limits $\dot{\boldsymbol{\beta}}_{\max }, \ddot{\boldsymbol{\beta}}_{\max }$ as well as avoiding singularities. This is quite a challenging objective given the complexity of such mobile robot structure [27], [28]. The output is the steer and drive joint velocity, corresponding to the feasible mobile base velocity vector $\dot{\mathbf{q}}_{b f}$. The framework in Fig. 4 consists of two decoupled controllers, one for steering (highlighted in red), and the other for driving (in blue). The former is responsible for achieving the direction of the desired robot velocity vector $\dot{\boldsymbol{\xi}}^{*}$ by acting on the steer joint configuration $\boldsymbol{\beta}$, and the latter is in turn responsible for the magnitude of $\dot{\boldsymbol{\xi}}^{*}$, by acting on the wheel velocity vector $\dot{\phi}$.

The controller uses the $3 D$ desired Cartesian robot velocity vector as input. This can be easily obtained from (3) by removing the zero vector $0_{3 \times 1}$ in the " $6 D$ to $3 D$ Cartesian Space Velocity Conversion" block in Fig. 4 The steering controller starts by mapping $\dot{\xi}^{*}$ to the $2 D$ ICR space $\boldsymbol{I C R ^ { * }}=\left[\begin{array}{ll}X^{*} & Y^{*}\end{array}\right]^{\top}$, where $X^{*}=-\dot{y}^{*} / \omega_{z}^{*}$, and $Y^{*}=\dot{x}^{*} / \omega_{z}^{*}$. The desired ICR point $\boldsymbol{I} \boldsymbol{C} \boldsymbol{R}^{*}$ is then modified based on the complementary route algorithm introduced in [?], which simply decides whether to move the current ICR point $\boldsymbol{I}$ C $\boldsymbol{R}_{\text {curr }}$ (corresponding to the current steer joint configuration) to the $\boldsymbol{I} \boldsymbol{C} \boldsymbol{R}^{*}$ following a direct or a

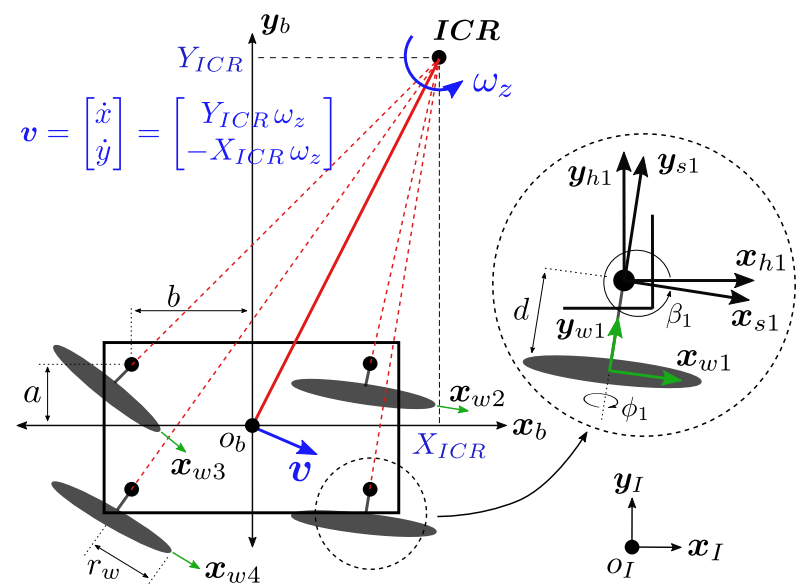

Fig. 3: Steerable wheeled mobile robot modeling schematic.

complementary route. The former is the direct straight line connecting the current and desired ICR points, while the latter is the shortest straight line connecting them, but moving in the opposite direction through the borders of the $2 D$ Cartesian ICR space. For more details, the reader is referred to [?].

The desired ICR motion $\boldsymbol{I} \boldsymbol{C} \boldsymbol{R}^{*}$, along with the current one, are fed to the ICR velocity controller which can be a simple proportional controller of gain $\lambda$ :

$$
\boldsymbol{I} \dot{\boldsymbol{C}} \boldsymbol{R}_{r e f}=\lambda\left(\boldsymbol{I} \boldsymbol{C} \boldsymbol{R}^{*}-\boldsymbol{I} \boldsymbol{C} \boldsymbol{R}_{\text {curr }}\right)
$$

The reference signal (output by numeric integration of the above) $\boldsymbol{I} \boldsymbol{C} \boldsymbol{R}_{r e f}$ is then used by a quadratic programming (QP) optimization algorithm to decide the "next sample time" ICR coordinates $\boldsymbol{I C} \boldsymbol{R}_{n e x t}$ that will minimize the quadratic cost error: $\left\|\boldsymbol{I} \boldsymbol{C} \boldsymbol{R}_{r e f}-\boldsymbol{I} \boldsymbol{C} \boldsymbol{R}_{n e x t}\right\|_{2}^{2}$ while respecting the joint performance limits formulated as linear constraints. For each steer joint, two straight lines are defined, the slopes of which are the maximum and minimum change in the steer joint variable in one sample period. These are related to $\dot{\boldsymbol{\beta}}_{\max }, \ddot{\boldsymbol{\beta}}_{\max }$. In the quadratic cost, $\boldsymbol{I} \boldsymbol{C} \boldsymbol{R}_{r e f}$ is used instead of $\boldsymbol{I C \boldsymbol { R } ^ { * }}$ to obtain a smooth behavior since the former is error dependent. The corresponding steer joint vector $\boldsymbol{\beta}_{\text {ref }}=\left[\begin{array}{lll}\beta_{1(r e f)} & \ldots & \beta_{4(r e f)}\end{array}\right]^{\top}$ is then evaluated using:

$$
\beta_{i(r e f)}=\arctan 2\left(Y_{\text {next }}-h_{y i}, X_{n e x t}-h_{x i}\right)-\pi / 2,
$$

where $i=1 \ldots 4$ for a 4 wheeled SWMR, and $h_{x i}$ and $h_{y i}$ denote the position of the $i^{t h}$ steer axis in the base frame. This is numerically differentiated, to obtain the $\dot{\boldsymbol{\beta}}_{r e f}$ that is sent to the robot low level controller.

The decoupled $3 D$ Cartesian space robot velocity controller produces an initial output $\dot{\boldsymbol{\xi}}_{\text {ref(init) }}$, computed by integrating $\ddot{\boldsymbol{\xi}}_{\text {ref(init })}=K_{p}\left(\dot{\boldsymbol{\xi}}^{*}-\hat{\dot{\boldsymbol{\xi}}}\right)$, where $K_{p}$ is a positive scalar gain. The controller output is then projected onto the null space of the "next sample time" kinematic constraint matrix to obtain the feasible control signal $\dot{\boldsymbol{\xi}}_{\text {ref }}$ that is compatible with the "next sample time" robot configuration. This is then converted to the $6 D$ vector $\dot{\mathbf{q}}_{b f}$ that is used by the motion controller in 15 . The reference wheel rate $\dot{\phi}_{\text {ref }}$ 


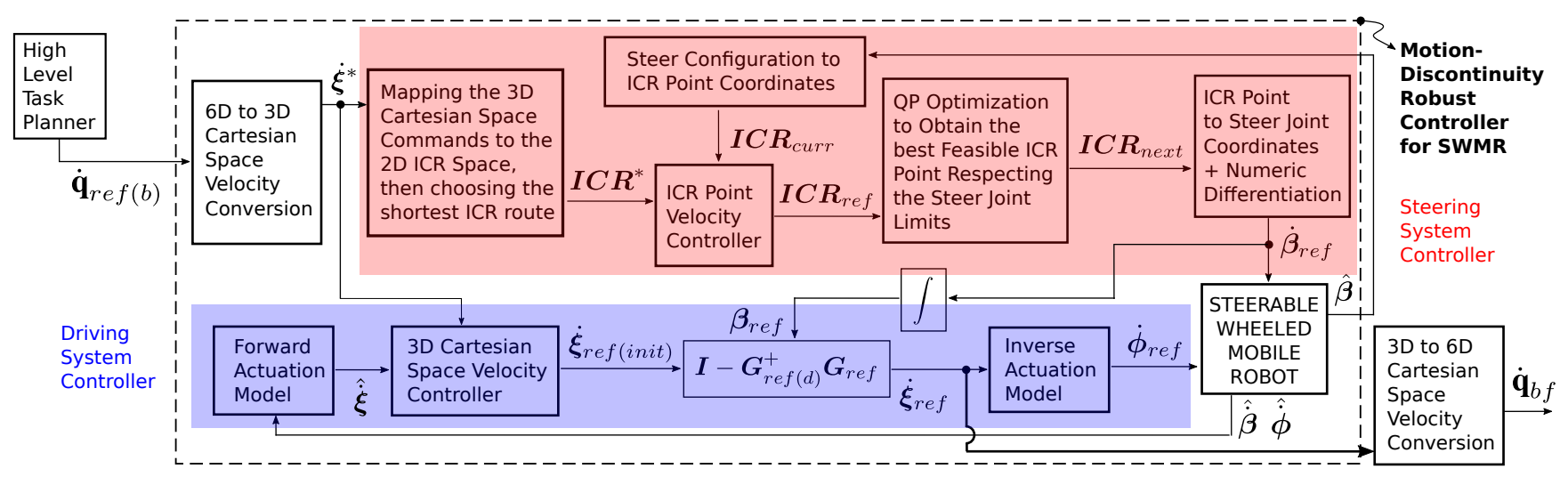

Fig. 4: Framework of the motion-discontinuity robust controller for steerable wheeled mobile robots.

is then obtained using the inverse actuation model:

$$
\begin{gathered}
\dot{\phi}_{i(r e f)}=\frac{1}{r_{w}} \boldsymbol{f}\left(\beta_{i(r e f)}\right) \dot{\boldsymbol{\xi}}_{\text {ref }}+\frac{d}{r_{w}} \dot{\beta}_{i(r e f)}, \\
\boldsymbol{f}(\beta)=\left[\begin{array}{lll}
\cos (\beta) & \sin (\beta) & d-h_{y i} \cos (\beta)+h_{x i} \sin (\beta)
\end{array}\right],
\end{gathered}
$$

where $d$ and $r_{w}$ denote the wheel offset and radius respectively. For more details about the framework and the kinematic model, the reader is referred to [23], [24], [29].

\section{Simulations}

To highlight the advantage of the proposed redundancy resolution in 9] and (14), we perform 3 simulation $11 \mathrm{em}-$ ploying first the whole body damped pseudo-inverse in (8), then method 9] without (second simulation) and with (third simulation) arm compensation (14.

\section{A. Setup}

All simulations are performed on the Vrep model of a mobile manipulator employing the Neobotix-MPO700 steerable wheeled mobile robot with a 7DOF KUKA LWR-IV arm, more details on the hardware setup can be found in [30], [31]. Simulation and controller parameters are provided in Table I where $t_{s}$ is the sampling period. In each simulation, the endtip is required to toggle between 5 different desired poses at distinct time instances. Details are provided in Table II where ${ }^{w} \mathbf{p}_{i}$ is the initial end-tip pose, the first 3 entries denote the position vector, measured in meters, and the remaining represent the RPY angles in radians. The first desired pose applied during the time period $t=[0,20[\mathrm{~s}$ is at relatively large distance from the initial end-tip state, requiring long travel in the workspace. Using the proposed controller, with or without arm compensation results in (as seen in Fig. 5-right) compact, predictable robot configuration while traversing towards such pose, a behavior highly favorable in human shared applications. On the contrary, using only the pseudo-inverse solution in (8) results in large mobile manipulation footprint (see Fig. 5-left) occupying much of space during such vast workspace travel. In Fig. 5, we can also observe that the proposed solution (9) results in more dextrous arm configuration as compared to (8).

\footnotetext{
${ }_{1}^{1}$ https://www.youtube.com/watch?v=FicvhIOIs $7 w \&$ feature=youtu.be
}

The second, third, and fourth poses applied during the periods $t=[20,25[\mathrm{~s}, t=[25,35[\mathrm{~s}$, and $t=[35,45[$ $\mathrm{s}$ respectively, feature small pose variation simulating a delicate manipulation task. Finally a relatively far desired pose is applied during $t=[45,65[\mathrm{~s}$ to verify the robustness of the mode switching (task sharing) function (11].

\section{B. Results}

The Euclidean norm of the pose error in 12 for the 3 simulations is shown in Fig. 6 The numbers on the respective

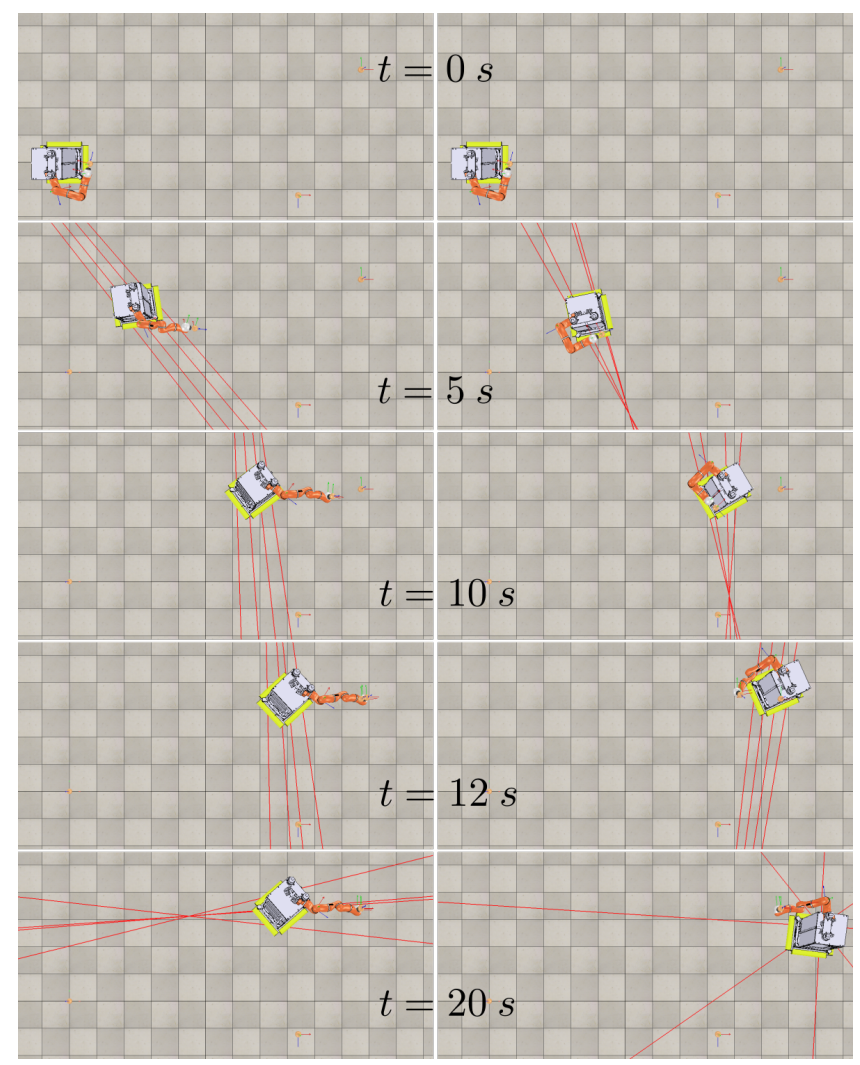

Fig. 5: Screen shots of the simulations featuring the proposed method (right) and the pseudo-inverse redundancy resolution (left) while moving from the initial pose to the first desired pose. It is obvious that the pseudo-inverse solution converges faster but with maximum footprint (base+arm) and higher error values as compared to the proposed solution. 

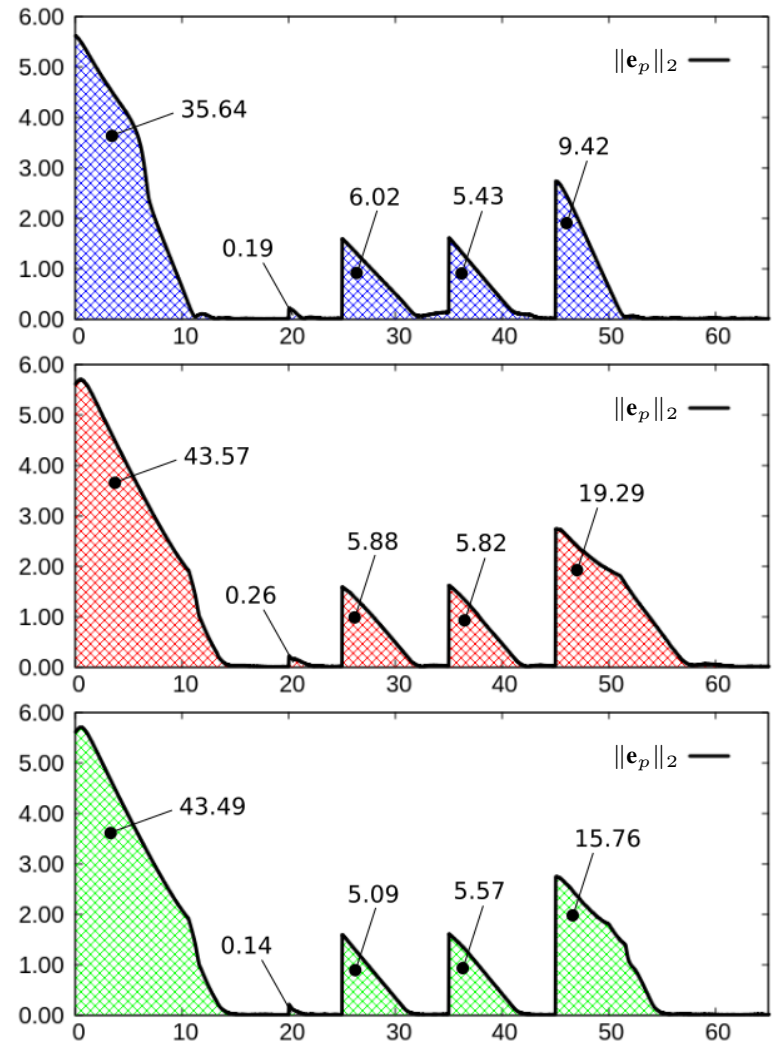

Fig. 6: Evolution of the Euclidean norm of pose error using the damped pseudo-inverse redundancy resolution (upper), the proposed method without arm compensation (middle), and the proposed with arm compensation. In all plots, the abscissa represents the simulation time instant (in seconds).

figure denote the area under the error curve of the pose control task for each of the desired poses. As shown in Fig. 6 (upper), the pseudo-inverse converges faster for the faraway poses since it generates the minimum Euclidean norm of total joint space velocities, resulting in the most time efficient motion. However, the large mobile manipulation footprint, along with all robot links motion, makes it less predictable and convenient for human-shared workspaces. The desired robot behavior is obtained using the proposed redundancy resolution in 9), although, applying arm compensation in (14), shown in Fig. 6(lower), enhances the error convergence

TABLE I: Simulation and controller parameters.

\begin{tabular}{|c||c||c||c||c||c|}
\hline$\lambda_{p}$ & 1.7 & $\lambda_{\text {dex }}$ & 3.5 & $n_{a}$ & 7 \\
\hline$r_{f m}$ & $0.25 m$ & $r_{m m}$ & $0.9 m$ & $\rho$ & 0.001 \\
\hline$r_{w}$ & $0.09 m$ & $t_{s}$ & $25 m s$ & $K_{p}$ & 2 \\
\hline$d$ & $0.045 m$ & $h_{x i}$ & $\pm 0.24 m$ & $h_{y i}$ & $\pm 0.19 m$ \\
\hline
\end{tabular}

TABLE II: Poses and configurations used.

\begin{tabular}{|c|c|}
\hline Pose/Configuration & Time Interval \\
\hline $\left.\begin{array}{lllll}0.004 & 1.08 & \pi / 2 & 0 & 0\end{array}\right]^{\top}$ & $t<0 s$ \\
\hline $\begin{array}{lll}1.5 & 1 & 0 \\
\end{array}$ & $t=[0,20[s$ \\
\hline${ }^{w} \mathbf{p}^{*}=[5$ & $t=[20,25[\mathrm{~s}$ \\
\hline${ }^{w} \mathbf{p}^{*}=\left[\begin{array}{llllll}4.8 & 1.3 & 1 & 0 & 0 & -\pi / 2\end{array}\right]^{\top}$ & $t=[25,35[s$ \\
\hline $\left.\begin{array}{llll}1 & 0 & 0 & 0\end{array}\right]^{\top}$ & $t=[35,45[s$ \\
\hline${ }^{w} \mathbf{p}^{*}=\left[\begin{array}{ll}4 & -0.5\end{array}\right.$ & $t=[45,65[\mathrm{~s}$ \\
\hline $\mathbf{q}_{a(\text { dex })}=\left[\begin{array}{llll}\frac{\pi}{2} & \frac{\pi}{2} & \frac{\pi}{2} & -\frac{\pi}{2}\end{array}\right.$ & $t=[0,65[s$ \\
\hline
\end{tabular}
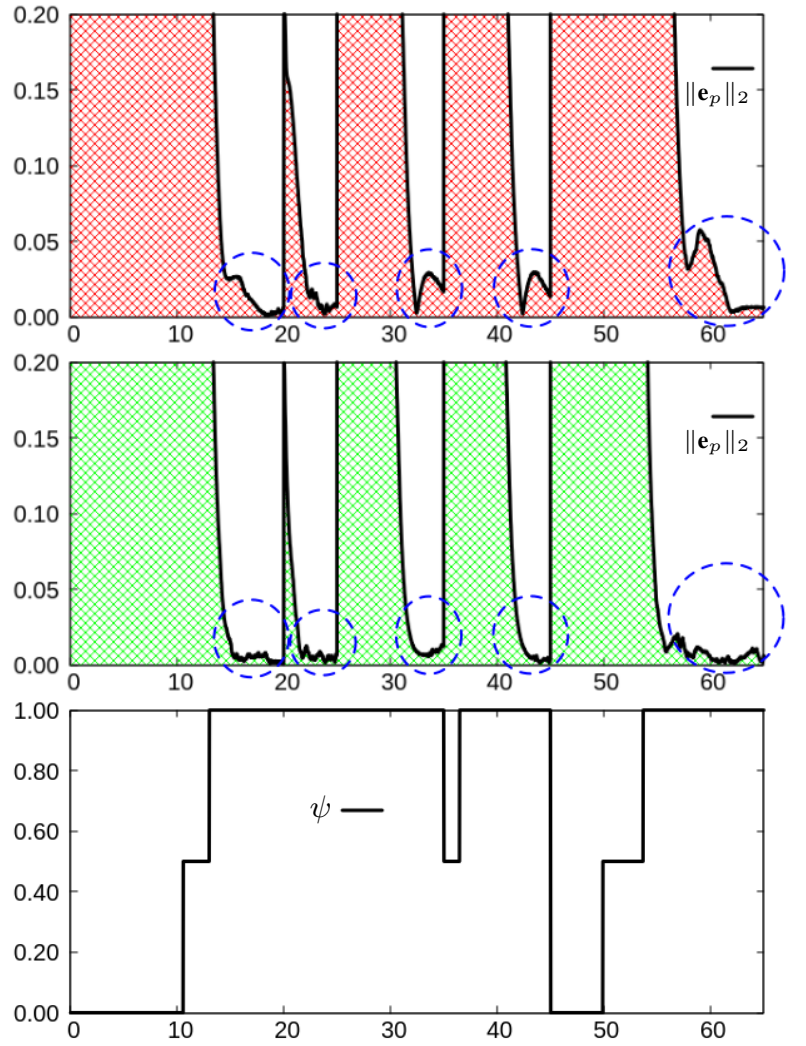

Fig. 7: A zoom in the pose error Euclidean norm for the proposed redundancy resolution without (upper) and with (middle) arm compensation, along with the task sharing parameter evolution for the former case. In all plots, the abscissa represents the simulation time instant (in seconds).

time as compared to Fig. 6 (middle).

Figure 7 gives a zoomed in view of the Euclidean norm error in Fig. 6 (middle), (lower). This figure depicts an additional advantage of using arm compensation, where the magnitude of steady state error is significantly smaller with better convergence compared to using (9) alone. Finally, Fig. 7 (lower) shows the evolution of the task sharing parameter $\psi$ in case of arm compensation. As expected, when reaching each desired pose, only the arm is activated $(\psi=1)$ to perform the pose control task. For the second and third desired poses, the arm is fully responsible since the desired change in pose is below the fine manipulation range $r_{f m}$.

\section{CONCLUSION}

We developed a redundancy resolution formula for steerable wheeled robots based mobile manipulation. The proposed method takes into account the feasible mobile robot velocity and accommodates for its inaccuracies firstly by decreasing its motion near the target pose and secondly by compensating its velocity error using the fast dynamics of the arm. Such error is predicted in advance thanks to the SWMR controller developed in our previous work. The proposed formula has been successfully validated in simulation.

\section{ACKNOWLEDGMENTS}

This work is supported by the French region Occitanie project CoBot@LR and by the PSA Robotics Department. It 
has also been supported by EPSRC under grant agreement EP/R02572X/1 (National Center for Nuclear Robotics).

\section{REFERENCES}

[1] H. Seraji, "A unified approach to motion control of mobile manipulators," The International Journal of Robotics Research, vol. 17, no. 2, pp. 107-118, 1998.

[2] A. D. Luca, G. Oriolo, and P. R. Giordano, "Kinematic modeling and redundancy resolution for nonholonomic mobile manipulators," in 2006 IEEE Int. Conf. on Robotics and Automation, May 2006, pp. $1867-1873$.

[3] E. Papadopoulos and J. Poulakakis, "Planning and model-based control for mobile manipulators," in 2000 IEEE/RSJ Int. Conf. on Intelligent Robots and Systems, vol. 3, 2000, pp. 1810-1815 vol.3.

[4] A. D. Luca, G. Oriolo, and P. R. Giordano, "Kinematic control of nonholonomic mobile manipulators in the presence of steering wheels," in 2010 IEEE Int. Conf. on Robotics and Automation, May 2010, pp. 1792-1798.

[5] R. Ancona, "Redundancy modelling and resolution for robotic mobile manipulators: a general approach," Advanced Robotics, vol. 31, no. 13, p. 706715, Jul 2017, dummy note.

[6] S. Sharma, G. K. Kraetzschmar, C. Scheurer, and R. Bischoff, "Unified closed form inverse kinematics for the kuka youbot," in ROBOTIK 2012; 7th German Conference on Robotics, May 2012, pp. 1-6.

[7] C. Scheurer, M. D. Fiore, S. Sharma, and C. Natale, "Industrial implementation of a multi-task redundancy resolution at velocity level for highly redundant mobile manipulators," in ISR 2016: 47st International Symposium on Robotics, June 2016, pp. 1-9.

[8] M. Mailah, E. Pitowarno, and H. Jamaluddin, "Robust motion control for mobile manipulator using resolved acceleration and proportionalintegral active force control," International Journal of Advanced Robotic Systems, vol. 2, no. 2, p. 14, 2005.

[9] L. Ellekilde and H. I. Christensen, "Control of mobile manipulator using the dynamical systems approach," in 2009 IEEE International Conference on Robotics and Automation, May 2009, pp. 1370-1376.

[10] A. Bhasin and A. Dutta, "Redundancy resolution and control of a nonholonomic mobile manipulator using kinect mounted on uav," in 2015 Conference on Advances In Robotics, no. 21. ACM, 2015, pp. $1-7$.

[11] N. A. M. Hootsmans and S. Dubowsky, "Large motion control of mobile manipulators including vehicle suspension characteristics," in 1991 IEEE International Conference on Robotics and Automation, April 1991, pp. 2336-2341 vol.3.

[12] H. Zhang, Y. Jia, N. Xi, and A. Song, "Obstacle avoidance for mobile manipulation by real-time sensor-based redundancy resolution," in 2012 IEEE International Conference on Robotics and Biomimetics (ROBIO), Dec 2012, pp. 2369-2374.

[13] V. Andaluz, F. Roberti, J. M. Toibero, and R. Carelli, "Adaptive unified motion control of mobile manipulators," Control Engineering Practice, vol. 20, no. 12, pp. 1337 - 1352, 2012.

[14] D. H. Shin, B. S. Hamner, S. Singh, and M. Hwangbo, "Motion planning for a mobile manipulator with imprecise locomotion," in 2003 IEEE/RSJ International Conference on Intelligent Robots and Systems (IROS 2003), vol. 1, Oct 2003, pp. 847-853 vol.1.

[15] T. Yoshikawa, "Manipulability of robotic mechanisms," The International Journal of Robotics Research, vol. 4, no. 2, pp. 3-9, 1985.

[16] B. Bayle, M. Renaud, and J.-Y. Fourquet, "Nonholonomic mobile manipulators: Kinematics, velocities and redundancies," Journal of Intelligent and Robotic Systems, vol. 36, no. 1, pp. 45-63, Jan 2003.

[17] B. Hamner, S. Koterba, J. Shi, R. Simmons, and S. Singh, "An autonomous mobile manipulator for assembly tasks," Autonomous Robots, vol. 28, no. 1, p. 131, Sep 2009.

[18] E. Simetti, G. Casalino, S. Torelli, A. Sperind, and A. Turetta, "Floating underwater manipulation: Developed control methodology and experimental validation within the trident project," Journal of Field Robotics, vol. 31, no. 3, pp. 364-385, 2014.

[19] Y. Nakamura, H. Hanafusa, and T. Yoshikawa, "Task-priority based redundancy control of robot manipulators," The International Journal of Robotics Research, vol. 6, no. 2, pp. 3-15, 1987.

[20] A. Mazur and D. Szakiel, "On path following control of nonholonomic mobile manipulators," International Journal of Applied Mathematics and Computer Science, vol. 19, no. 4, pp. 561-574, 2009.

[21] J. S. Chipman, "On least squares with insufficient observations," Journal of the American Statistical Association, vol. 59, no. 308, pp. 1078-1111, 1964.
[22] B. Navarro, A. Cherubini, A. Fonte, G. Poisson, and P. Fraisse, "A framework for intuitive collaboration with a mobile manipulator," in 2017 IEEE/RSJ International Conference on Intelligent Robots and Systems, 2017.

[23] M. Sorour, A. Cherubini, P. Fraisse, and R. Passama, "Motion discontinuity-robust controller for steerable mobile robots," IEEE Robotics and Automation Letters, vol. 2, no. 2, pp. 452-459, April 2017.

[24] M. Sorour, A. Cherubini, A. Khelloufi, R. Passama, and P. Fraisse, "Complementary-route based icr control for steerable wheeled mobile robots," Robotics and Autonomous Systems, 2019. [Online]. Available: http://www.sciencedirect.com/science/article/pii/S0921889018305086

[25] Y. Nakamura and H. Hanafusa, "Inverse kinematic solutions with singularity robustness for robot manipulator control," ASME Journal of Dyn. Sys., Measur., and Control, vol. 108, no. 3, pp. 163-171, 1986.

[26] C. W. Wampler, "Manipulator inverse kinematic solutions based on vector formulations and damped least-squares methods," IEEE Trans. on Sys., Man, and Cybernetics, vol. 16, no. 1, pp. 93-101, 1986.

[27] C. Stoger, A. Muller, and H. Gattringer, "Kinematic analysis and singularity robust path control of a non-holonomic mobile platform with several steerable driving wheels," in 2015 IEEE/RSJ International Conference on Intelligent Robots and Systems, 2015, pp. 4140-4145.

[28] L. Clavien, M. Lauria, and F. Michaud, "Instantaneous centre of rotation based motion control for omnidirectional mobile robots with sidewards off-centred wheels," Robotics and Autonomous Systems, vol. 106, pp. $58-68,2018$.

[29] M. Sorour, A. Cherubini, R. Passama, and P. Fraisse, "Kinematic modeling and singularity treatment of steerable wheeled mobile robots with joint acceleration limits," in IEEE Int. Conf. on Robotics and Automation, 2016, pp. 2110-2115.

[30] A. Cherubini, A. Crosnier, P. Fraisse, B. Navarro, R. Passama, and M. Sorour, "Research on cobotics at the lirmm idh group," ICRA Workshop IC3 Industry of the future, 2017.

[31] A. Cherubini, R. Passama, B. Navarro, M. Sorour, A. Khelloufi, O. Mazhar, S. Tarbouriech, J. Zhu, O. Tempier, A. Crosnier, P. Fraisse, and S. Ramdani, "A collaborative robot for the factory of the future: Bazar," The International Journal of Advanced Manufacturing Technology, May 2019. 